\title{
JUURNAL.RU
}

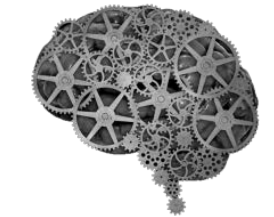

COMPANY GROUP "INTELLEKT"

Разумов И.А. Ульяновский государственный университет

Ульяновск, Россия

doi: 10.18411/lj2016-6-3-08

\section{Построение модели продуктовой политики научно-производственного объединения}

В настоящее время в связи с объективно обусловленным усложнением значительного числа промышленных изделий наблюдается соответствующее усложнение технологических и организационных основ производства. Как следствие, осознается потенциальная эффективность таких интегрированных производственных структур как научно-производственные объединения, что требует разработки и внедрения в практику управления новых методов принятия решений. Одним из аспектов управления интегрированными производственными структурами является формирование и реализация продуктовой политики, которую следует понимать как некий шаблон производственного плана и, одновременно, как процедурный стереотип. Традиционно продуктовая политика относится к сфере маркетинговых методов и средств. Примером тому может служить известная публикация [1]. Но в настоящее время по объективным причинам проблематику, связанную с продуктовой политикой целесообразно распространить и на другие области, прежде всего, на задачи управления производственными объектами. Разумеется, все решения, которые связаны с продуктовой политикой, основаны на определенной модели. Очевидно, что особенности производства продуктов, свойственные различным отраслям промышленности, могут существенно повлиять на форму такой модели. Наиболее адекватной формой модели в 
данном случае, как представляется, может быть только теоретикомножественная модель продуктовой политики.

Спецификация модели продуктовой политики основана на результатах логического анализа проблемной ситуации. Очевидно, что наиболее приоритетным компонентом модели в данном случае следует считать потребности технической среды, на удовлетворение которых направлена деятельность интегрированной производственной системы. То есть, одним из основных компонентов модели продуктовой политики следует считать множество R:

$$
\mathbf{R}=\left(\mathrm{R}_{1}, \mathrm{R}_{2}, \ldots, \mathrm{R}_{\mathrm{n}}, \mathrm{r}_{1}, \mathrm{r}_{2}, \ldots, \mathrm{r}_{\mathrm{m}}\right)
$$

где Ri - это продукция авиаприборостроительных предприятий, которая востребована потенциальными потребителями.

rj - это сборочные единицы функционально полных комплексов или же изделия меньшего уровня сложности, а также изделия, востребованные в других отраслях промышленности или же входящие в состав сервисных комплектов.

Элементы множества $\mathrm{R}$ обуславливают формирование устойчивых коммерческих и кооперационных связей, требующих того, чтобы их учли в продуктовой политике и, соответственно, в модели, предполагающей использование в процессе принятия управленческих решений. Очевидно, что множество R представляет собой достаточно обширный список продукции классификатор перечислительного типа.

Представляется, что все элементы формальных моделей должны определенным способом интерпретироваться. Содержательный аспект абстрактных моделей представляет собой основное средство обеспечения их адекватности.

Для определения смысловой составляющей выражения классификаторы высокого уровня, такие как ОКП непосредственно использоваться вряд ли могут в силу формы представления информации. Таким образом, состав этого множества может быть задан имплицитно, а для 
практиков, вынужденных каким-то образом учитывать этот состав в своих умозаключениях, имплицитное задание элементов этого множества целесообразно сопроводить примерами, иллюстрирующими основные направления развития технической среды, в которой функционирует интегрированная производственная структура, формирующая и реализующая продуктовую политику.

В первую очередь обращает на себя внимание особенность перечисленных групп продукции, которая, как представляется, может определенным образом оказать влияние на результаты как теоретического анализа, так и практических решений, получаемых с использованием принципов и положений, сформулированных в ходе разработки модели, причем, эта особенность представляется неочевидной, и не может быть выявлена в процессе разработки модели проблемной ситуации. Речь идет о том, что перечисленные группы продукции отрасли соответствуют принципу аналогичности-дополнения. В самом деле, многие различные изделия содержат в своем составе детали и агрегаты общего назначения, что является существенным именно для продуктовой политики интегрированных производственных структур.

В неявном виде для всех перечисленных выше продуктовых групп техническая среда, выступающая в качестве заказчика и потенциального потребителя продукции предприятий отрасли, исследованной в процессе подготовки настоящей работы, выдвигает показатели, по которым можно оценивать производственный потенциал различных предприятий и организаций. К основным таким показателям относятся следующие:

- функционал или показатели назначения, что характеризует в первую очередь специализацию проектировщиков, входящих в состав интегрированных производственных структур, таких как научнопроизводственные объединения;

- надежность и отказоустойчивость;

- массо-габаритные характеристики; 
- уровень энергопотребления;

- стоимость;

- удобство эксплуатации;

- ремонтопригодность, в том числе наличие развитой системы сервисного обслуживания.

Следует обратить внимание, что требования, выдвигаемые технической средой, формируются в условиях достаточно жесткой конкурентной борьбы с мировыми производителями продуктов-аналогов.

Компонентом модели продуктовой политики, описывающим технологический потенциал интегрированной производственной структуры, является множество Т:

$$
\mathbf{T}=\left(\mathrm{t}_{\mathrm{i} 1}, \mathrm{t}_{\mathrm{i} 2}, \ldots \mathrm{t}_{\mathrm{ik}}, \mathrm{t}_{\mathrm{d} 1}, \mathrm{t}_{\mathrm{d} 2}, \ldots, \mathrm{t}_{\mathrm{d} 1}, \mathrm{t}_{\mathrm{a} 1}, \mathrm{t}_{\mathrm{a} 2}, \ldots . \mathrm{t}_{\mathrm{am}}\right)
$$

где tij - информационные компоненты технологического обеспечения производственного процесса;

$\mathrm{tdz}$ - основные средства, используемые в производстве определенного вида продукции;

tav - оснастка, инструмент и приспособления, используемые в производстве определенного вида продукции.

Семантика этого компонента модели продуктовой политики при имплицитном её задании может быть проиллюстрирована данными, характеризующими организации, которые можно считать типовыми представителями отрасли. Именно эти организации явились участниками интеграционных процессов, в результате которых большая часть из них поменяли статус, войдя в состав научно производственного объединения.

Еще одним компонентом модели продуктовой политики является множество организационных, в первую очередь кадровых ресурсов О:

$$
\mathbf{O}=\left(\mathrm{o}_{\mathrm{p} 1}, \mathrm{o}_{\mathrm{p} 2}, \ldots \mathrm{o}_{\mathrm{ps}}, \mathrm{o}_{\mathrm{e} 1}, \mathrm{o}_{\mathrm{e} 2}, \ldots \mathrm{o}_{\mathrm{eu}}, \mathrm{o}_{\mathrm{su} 1}, \mathrm{o}_{\mathrm{su} 2} \ldots \mathrm{o}_{\mathrm{suy}}\right)
$$

где орј - показатель, характеризующий производственный персонал определенной квалификации и специальности; 
оеі - показатель, характеризующий инженерный персонал;

osuk - показатели, характеризующие систему управления.

Теоретический взгляд на проблему описания продуктовой политики позволяет сделать следующее допущение. Объединение технологического и кадрового потенциала делает возможным включение в модель реализуемых вариантов продуктовой политики, что в формальном виде можно представить следующим образом:

$$
\mathbf{O} \times \mathbf{T}=\mathbf{R}_{\text {real }} \subseteq \mathbf{R}
$$

где Rreal - потенциально реализуемые варианты продуктовой политики в плане организационно-технологических возможностей интегрировано производственной системы. Данное подмножество входит составной частью в множество R.

Очевидно, что подмножество Rreal представляет собой один из вариантов продуктовой политики, который складывается на эмпирической основе и является результатом специализации, сформированной в силу многих факторов, трудно поддающихся учету. Кроме того, данный вариант продуктовой политики не содержит процедурного компонента, поскольку организации действуют как самостоятельные субъекты хозяйствования. Развивая данное положение, можно прийти к следующему выводу.

Объединение производственных возможностей в виде варианта Rreal позволит усилить организациям позиции на рынке, повышая эффективность хозяйствования за счет фактора диверсификации. Кроме того, объединение ресурсов за счет возможности гибкого реагирования на колебания конъюнктуры и манипулирования имеющимися ресурсами сформирует базу для использования резервов интегрированных структур.

Для создания подобных возможностей в модели должны быть предусмотрены такие компоненты как финансовое обеспечение и критерии, под которыми понимаются методы и средства оценки ситуации и принятия решений. Наличие финансовых ресурсов F позволит множество Rreal реализуемых 
вариантов сузить до набора вариантов приемлемых или даже оптимальных в смысле набора критериев принятия решения К:

$$
\mathbf{R}_{\text {opt }} \subseteq \mathbf{R}_{\text {real }}
$$

Если предшествующие выражения можно было отнести к шаблонам производственных планов научно-производственного объединения, то компонент модели, соответствующий финансовому обеспечению и используемым критериям принятия управленческих решений можно поставить в соответствие процедурному аспекту продуктовой политики.

Таким образом, общая модель продуктовой политики интегрированной производственной системы будет выглядеть следующим образом:

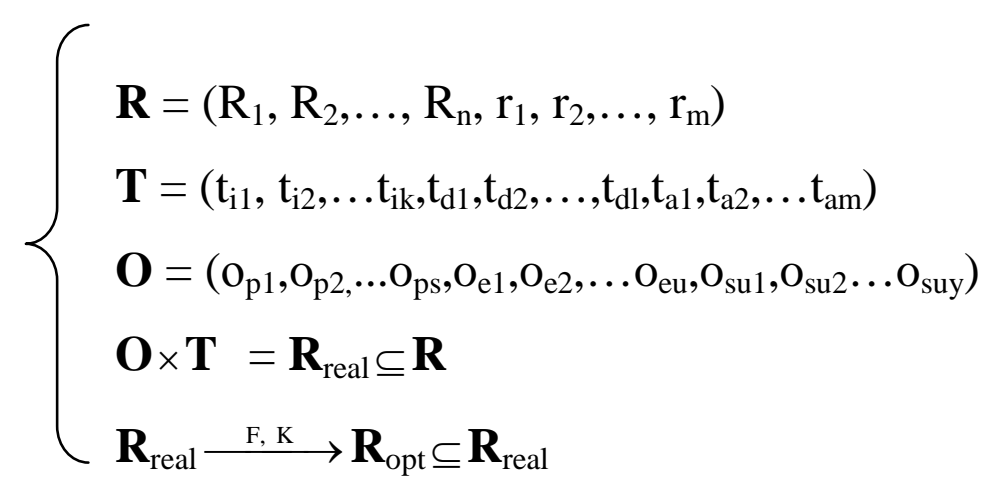

Модель продуктовой политики, представленная в виде (6), как представляется, может быть использована в различных процессах подготовки и принятия решений, прежде всего, для организации экспертных процедур. 


\section{Список используемых источников информации}

1. Голубков Е.П. Проектирование элементов комплекса маркетинга: формирование продуктовой политики / Маркетинг в России и за рубежом

- 2001, №5.[Электронный ресурс]. /Е.П.Голубков // Режим доступа: http://www.mavriz.ru/articles/2001/5/353.html (дата обращения: 02.09.2016). 